\title{
Exclude Vital Signs Data From Statistics Indicator
}

National Cancer Institute

\section{Source}

National Cancer Institute. Exclude Vital Signs Data From Statistics Indicator. NCI

Thesaurus. Code C119949.

Specifies whether the vital signs result values should be excluded from the statistical analysis. 